Fernández Noguerol, S. (2017): "Desarrollo de herramientas para el tratamiento de la información y el análisis con SIG de los usos del suelo utilizando el SIOSE. Una aproximación al caso de Asturias.”, GeoFocus (Artículos), $n^{\circ} 20, p .233-$ 251. ISSN: $1578-5157$ http://dx.doi.org/10.21138/GF.573

\title{
DESARROLLO DE HERRAMIENTAS PARA EL TRATAMIENTO DE LA INFORMACIÓN Y EL ANÁLISIS CON SIG DE LOS USOS DEL SUELO UTILIZANDO EL SIOSE. UNA APROXIMACIÓN AL CASO DE ASTURIAS.
}

\author{
SANTOS FERNÁNDEZ NOGUEROL ${ }^{1}$ \\ ${ }^{1}$ Departamento de Geografía. Universidad de Oviedo \\ c/ Amparo Pedregal s/n, 33011 Oviedo, España \\ santos@gistools.es
}

\section{RESUMEN}

Se describe el desarrollo de un programa capaz de descifrar el código de cada polígono del SIOSE. El objetivo es recabar todos los datos que puedan obtenerse de los rótulos y presentar los resultados tabulados para facilitar y ampliar considerablemente el manejo y las posibilidades de análisis de la fuente. Además, con la intención de demostrar la utilidad práctica, se ha empleado la información conseguida para realizar un análisis geográfico breve sobre los usos del suelo de Asturias que ayuda al estudio de los tipos de paisaje presentes en la región.

Palabras clave: SIOSE, usos del suelo, paisaje, Asturias, sistemas de información geográfica, SIG.

\section{DEVELOPING TOOLS FOR DATA MANAGEMENT AND ANALYSIS OF LAND USES THROUGH SIOSE AND GIS. AN APPROACH TO THE CASE OF ASTURIAS.}

\begin{abstract}
It is described the development of a program able to decrypt the code of each SIOSE polygon. The aim is to collect all the data that can be taken from the labels and present the results tabulated to ease and improve remarkably the management and the analysis possibilities of this source. In addition, with the purpose of demonstrating the practical usefulness, the information obtained has been used to conduct a brief geographical analysis of Asturias that, based on land uses, helps to study the types of landscapes of the region.
\end{abstract}

Keywords: SIOSE, land uses, landscape, Asturias, geographic information systems, GIS. 
Fernández Noguerol, S. (2017): "Desarrollo de herramientas para el tratamiento de la información y el análisis con SIG de los usos del suelo utilizando el SIOSE. Una aproximación al caso de Asturias.”, GeoFocus (Artículos), $n^{\circ} 20, p .233-$ 251. ISSN: $1578-5157$ http://dx.doi.org/10.21138/GF.573

\section{Introducción}

A comienzos de 2012 el Instituto Geográfico Nacional (IGN) hizo público el "Sistema de Información sobre Ocupación del Suelo de España" (SIOSE) con datos de referencia de 2005, publicándose en 2016 la actualización de 2011. Durante varios meses, la primera versión fue distribuida a través del Centro de Descargas de Centro Nacional de Información Geográfica (CNIG) aportando: copia del SIOSE (un Shapefile con la representación gráfica y una base de datos Access con la información alfanumérica) y de la documentación técnica que explica la estructura de la fuente. No existían o no se podía acceder a herramientas que facilitasen a los usuarios la explotación de los datos, codificados con un nivel de detalle sin precedentes.

A finales de 2012 esta situación cambió sustancialmente al hacerse público el programa "SIOSE Desktop", cuyo desarrollo correspondió a la empresa pública Tragsatec. La herramienta, difundida desde entonces del mismo modo que los datos, permite realizar consultas, visualizar gráficos de síntesis y exportar los resultados a distintos formatos de archivo. En definitiva, gracias a ella se facilitaron considerablemente las posibilidades de análisis.

El desarrollo del programa que se presenta en este artículo discurrió de forma paralela a los hechos anteriores y estuvo motivado por las necesidades surgidas durante la realización de la tesis doctoral titulada "Transformaciones territoriales recientes en el valle del Caudal (Asturias, España) como consecuencia de la reconversión industrial". A fin de incorporar al trabajo la cartografía de usos del suelo más reciente, y ante la carencia de utilidades que facilitasen la tarea en aquel momento, fue necesario diseñar una aplicación propia que permitiese descifrar el código SIOSE de cada polígono (Fernández Noguerol, 2015).

Tras una revisión del estado de la cuestión, dónde se describirán las características de la fuente y se revisarán los principales estudios publicados sobre la cuestión, se procederá a describir la metodología empleada. Posteriormente, se explicará el algoritmo que descifra el código SIOSE y, para valorar su utilidad, se desarrollará un método de análisis geográfico aplicado a un caso concreto. Antes de abordar las conclusiones, se presentarán los resultados obtenidos, tanto en lo concerniente a los datos en bruto, como a la plasmación espacial de los mismos.

\section{Estado de la cuestión}

\subsection{Características de los datos del SIOSE}

Según puede consultarse en la documentación técnica, el polígono es la unidad de trabajo, siendo la única entidad con geometría delimitada y usos del suelo homogéneos. Dentro de él se inscriben los conceptos teóricos de uso y cobertura. El primero de ellos no tiene implicación práctica en el modelo de datos actual al haber sido incluido exclusivamente de modo formal con vistas a desarrollarlo en el futuro. La segunda es considerada como un espacio continuo del terreno con un conjunto de atributos que la caracterizan y representa el $100 \%$ de la superficie de cada polígono, siendo simple cuando no puede descomponerse en otras y compuesta cuando está formada por dos o más simples y/o compuestas (IGN, 2015).

Para representar el tipo ocupación que tiene un polígono se utiliza un código que recibe el nombre de "rótulo SIOSE". En él están representadas todas las coberturas que lo definen que, en aquellos casos más complejos, tienen la siguiente representación: PPCCCaa1aa2 (PP: porcentaje de 
Fernández Noguerol, S. (2017): "Desarrollo de herramientas para el tratamiento de la información y el análisis con SIG de los usos del suelo utilizando el SIOSE. Una aproximación al caso de Asturias.”, GeoFocus (Artículos), $n^{\circ} 20, p .233-$ 251. ISSN: $1578-5157$ http://dx.doi.org/10.21138/GF.573

ocupación si es menor del $100 \%$, CCC: etiqueta de la cobertura, aa1: primer atributo y aa2: segundo atributo). Cuando los polígonos están definidos por más de una cobertura, el rótulo emplea “" para separar cada una de ellas y "( )" para subdefinir las coberturas compuestas.

En total existen 85 coberturas (40 simples y 45 complejas), así como 23 atributos (subdivididos a su vez en distintos tipos). Todo ello genera un modelo muy detallado que, por otra parte, dificulta la explotación de los datos. Hasta la fecha, la denominada "tabla plana" incluida en una base de datos Access y el programa "SIOSE Desktop" constituyen las herramientas que el IGN ha hecho públicas para trabajar la información. Ambas tienen gran utilidad y nivel de desagregación, al fundamentarse en las etiquetas y sus valores asociados para realizar consultas con las que, por ejemplo, se pueden generar mapas de coropletas a posteriori.

Sin embargo, se han obviado varias clasificaciones que la documentación técnica recoge y que aumentan las posibilidades de explotación analítica. Estas son las principales: tipo, tema y subtema. En el caso del tipo, una cobertura puede ser "simple", "compuesta" y "predefinida". El tema puede ser "cultivo", "forestal" o "artificial compuesto", entre otros. El subtema abarca valores como "cultivos herbáceos", "frondosas", "industrial" ...

Si bien es cierto que las distintas agrupaciones señaladas pueden conseguirse de forma manual agregando los datos con la "tabla plana" o "SIOSE Desktop", en la práctica ello puede conllevar errores como consecuencia de lo tediosa que puede resultar la tarea, puesto que deben cotejarse numerosas coberturas. Tampoco ayuda el hecho de que, en la mayoría de los supuestos, la misma acción debe repetirse varias veces para poder establecer comparaciones entre los distintos usos del suelo.

\subsection{Publicaciones y ejemplos de análisis desarrollados hasta la fecha}

Desde que se inició la difusión pública del SIOSE han aparecido tres grandes tipos de publicaciones: documentos técnicos, materiales de difusión y estudios que aprovechan los datos con distintos fines. Los primeros han sido elaborados fundamentalmente por el IGN y pueden consultarse en la página web del proyecto (IGN, 2017); su función es la de explicar el proceso de elaboración, el control de calidad, la estructura de las coberturas y los metadatos. Además, se ha realizado alguna publicación técnica en el extranjero para dar a conocer la fuente, siendo la más destacable la presentada a la Sociedad Internacional para la Fotogrametría y la Teledetección (Valcarcel et al., 2008). También ha habido algún autor que ha tratado de elaborar un modelo jerárquico para el SIOSE que permita utilizarlo más fácilmente, proponiendo una estructura de datos similar a la del Corine Land Cover (Cantarino Martí, 2013).

El segundo grupo también tiene un número reducido de publicaciones. La primera de ellas apareció en el "XI Congreso nacional de Teledetección" celebrado en 2005 en el Puerto de La Cruz (Tenerife), cuando el IGN presentó una ponencia en la que se describían las principales características del proyecto (Del Bosque González et al., 2005). Posteriormente y en un contexto similar, durante el "XIII Congreso nacional de Tecnologías de la Información Geográfica" celebrado en 2008 en Las Palmas de Gran Canaria, concurrieron tres ponencias relacionadas: una sobre la elaboración de la fuente utilizando Geomedia (Rubio Díaz et al., 2008) y dos sobre la experiencia de Andalucía (Moreira et al., 2008) y Cataluña (Burriel Moreno et al., 2008) en la confección del SIOSE. En fechas más recientes, una presentación del IGN explicaba la 
Fernández Noguerol, S. (2017): "Desarrollo de herramientas para el tratamiento de la información y el análisis con SIG de los usos del suelo utilizando el SIOSE. Una aproximación al caso de Asturias.”, GeoFocus (Artículos), $n^{\circ} 20, p .233-$ 251. ISSN: $1578-5157$ http://dx.doi.org/10.21138/GF.573

generalización del SIOSE para producción del Corine Land Cover de 2012 mediante el uso de FME y ArcGIS (Delgado Hernández, 2015).

El tercer grupo es el más amplio y diverso de los tres ya que, teniendo en cuenta lo comentado en el apartado anterior, cada autor ha optado por distintas metodologías para obtener provecho de la fuente. Siguiendo un criterio de clasificación temático, varias materias han captado el esfuerzo de los autores: el medio urbano, el rural y los estudios generales de síntesis sobre una Comunidad Autónoma o región concreta.

Los que toman a las ciudades como campo de trabajo han utilizado como escala de referencia la regional en la mayoría de los casos, destacando uno sobre las capitales valencianas (Martí-Ciriquián et al., 2014) y otro sobre los espacios periurbanos en Murcia (Rosa Fernández et al., 2013). También hay algún ejemplo a nivel inframunicipal que se centra en el análisis de la estructura urbana mediante bases de datos detalladas (Santos Preciado et al., 2014). Junto a los anteriores, una investigación publicada por la Fundación BBVA valora la morfología urbana a nivel estatal (Goerlich Gisbert et al., 2013).

Los espacios rurales de España fueron estudiados también por Goerlich Gisbert (2013), quién repasó las características de las coberturas del suelo, con la idea de explotar el SIOSE como una fuente de información para el establecimiento de una tipología rural/urbana a nivel municipal y al margen de los aspectos demográficos. Complementariamente, siguiendo un enfoque agrícolaforestal a escala provincial, en La Rioja fue analizada la diversidad vegetal de los campos abandonados del Leza y Jubera (Lasanta et al., 2011).

Por regiones hay ejemplos de Galicia (Díaz Manso et al., 2007), Tenerife (Pérez Hernández, 2015), Andalucía (Alcántara et al., 2014)... Entre estas últimas se incluye Asturias que, además de ser tomada como objeto de estudio en este artículo, fue abordada en un trabajo de fin de master realizado en la Universidad de Oviedo (Rodríguez Gozalo, 2012). A nivel estatal destaca una publicación surgida en la Universitat Autònoma de Barcelona en la que se combina el SIOSE con imágenes Landsat para obtener automáticamente cartografía retroactiva (Vidal-Macua et al., 2017).

\section{Metodología}

La herramienta planteada tiene como objetivo descifrar los rótulos del SIOSE contenidos en el shapefile de polígonos distribuido por el IGN e incorporado a una geodatabase, generando por cada uno de ellos, tantas copias del mismo como coberturas lo definan. De esta manera, si un polígono está compuesto por tres usos, en la clase resultante se generarán tres entidades superpuestas conformes a la original, teniendo cada una de ellas los valores de una sola cobertura y siguiendo el orden de aparición en el rótulo.

Para conseguirlo, dado que los principales Sistemas de Información Geográfica (SIG) se ejecutan en sistemas operativos Windows, se optó por utilizar la plataforma ".NET" de Microsoft, utilizando en este caso el lenguaje C\# para escribir el código fuente y poder utilizar "ArcObjects 10.5" de ESRI, paquete de desarrollo de software que permitió integrar la herramienta en ArcGIS. 
Fernández Noguerol, S. (2017): "Desarrollo de herramientas para el tratamiento de la información y el análisis con SIG de los usos del suelo utilizando el SIOSE. Una aproximación al caso de Asturias.”, GeoFocus (Artículos), $n^{\circ} 20, p .233-$ 251. ISSN: $1578-5157$ http://dx.doi.org/10.21138/GF.573

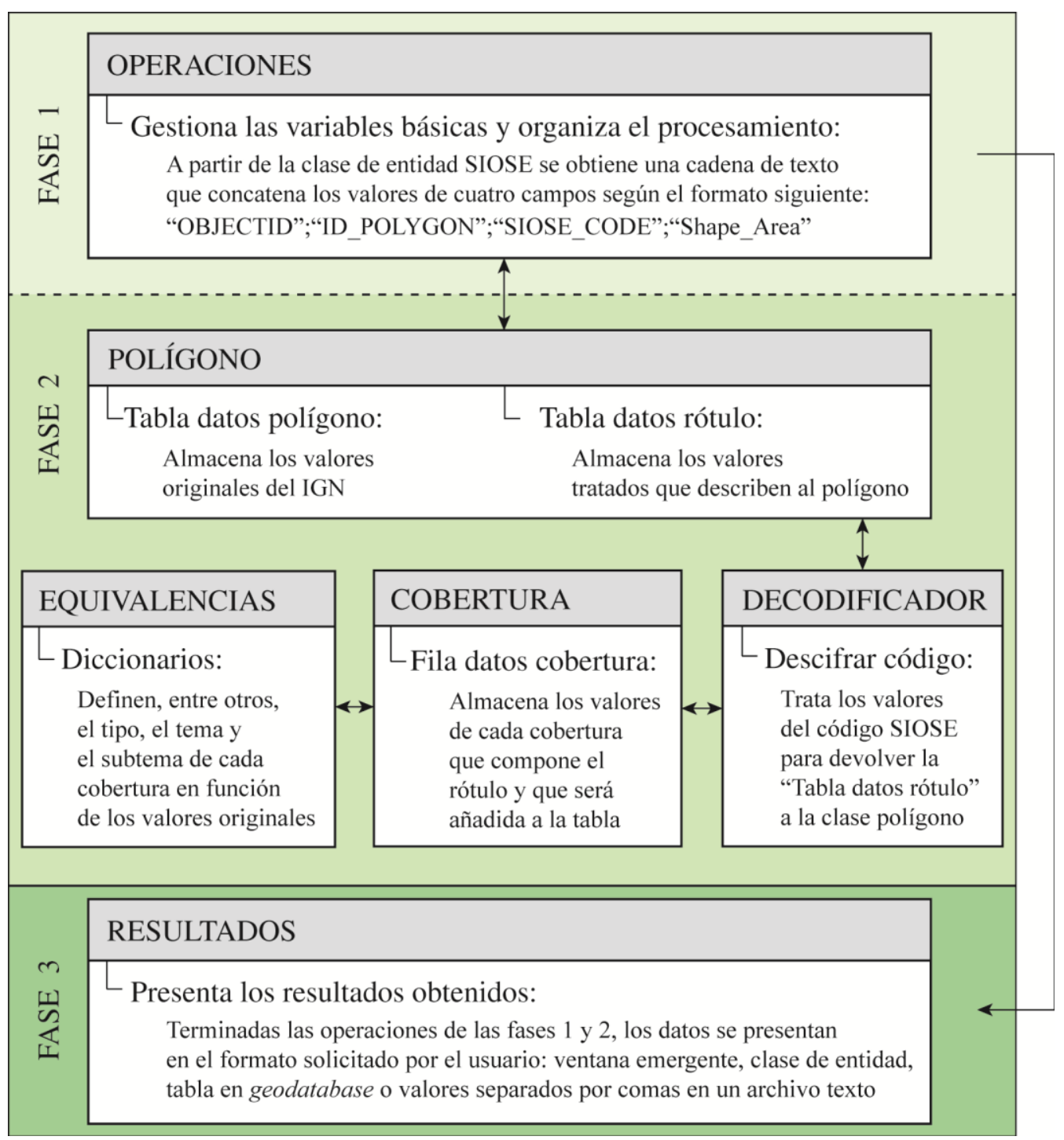

Figura 1. Diagrama de síntesis que describe la organización y el proceso de ejecución del algoritmo de la herramienta para descifrar el código SIOSE.

Fte. Elaboración propia.

Como puede verse en la figura 1, la estructura del programa está compuesta principalmente por cinco elementos diferenciados o clases. El usuario solo puede acceder directamente a la denominada "operaciones", cuya función principal es la de inicializar los parámetros básicos necesarios que permiten alcanzar los objetivos, además de organizar el proceso en función del formato de datos en el que se desea presentar el resultado, pudiendo ser: ventanas emergentes, tablas o clases de entidad en geodatabases o archivos de texto (txt). Durante la segunda fase, la siguiente clase de referencia se llama "polígono" y es la encargada de almacenar en memoria la información original y derivada de cada entidad geográfica representada en el SIOSE. En ella se guardan en dos tablas temporales los datos originales suministrados por el IGN y los valores 
Fernández Noguerol, S. (2017): "Desarrollo de herramientas para el tratamiento de la información y el análisis con SIG de los usos del suelo utilizando el SIOSE. Una aproximación al caso de Asturias.”, GeoFocus (Artículos), $n^{\circ} 20, p .233-$ 251. ISSN: $1578-5157$ http://dx.doi.org/10.21138/GF.573

descifrados que son obtenidos en el "decodificador". Esta última clase toma el código del campo "SIOSE_CODE" de cada polígono para leerlo secuencialmente, obteniendo a partir de él las coberturas que lo integran, los atributos y el porcentaje de ocupación. Durante la segunda fase, con la etiqueta que define el uso del suelo se obtienen también las equivalencias y las clasificaciones definidas en la documentación técnica, siendo las encargadas de ambas tareas las clases auxiliares "cobertura" y "equivalencias" (IGN, 2015). Cuando el decodificador concluye, devuelve una lista de objetos "polígono" con toda la información a la clase "operaciones". Comienza entonces la tercera y última fase, dedicada a almacenar en el disco o mostrar en pantalla los datos procesados.

Habiendo finalizado el tratamiento de la fuente original, se buscó dar aplicación a los resultados mediante el planteamiento de un análisis que podría denominarse como "perspectiva paisajística". Para ello se optó por utilizar ArcGIS ya que dispone de las herramientas necesarias para llevar a cabo el estudio. Con la intención de automatizar el procedimiento y para evitar errores, se escribió un script con "ArcPy" en el que, entre otras, se realizan dos tareas fundamentales. La primera es un análisis de visibilidad que tiene en cuenta un punto concreto en el espacio, cuyo resultado es utilizado en segunda instancia para recortar la capa de usos del suelo del SIOSE obtenida tras la ejecución de la herramienta de descifrado. Cuando termina la operación, se presenta la extensión de terreno visible desde un lugar determinado y las ocupaciones que intersecta.

Para poner en práctica el análisis de la perspectiva paisajística y tras realizar diversas pruebas con varias regiones de España, se ha optado por seleccionar como ámbito de estudio al Principado de Asturias. Esa decisión se debe, en primer lugar, a que se encontraron rótulos más complejos que en otras regiones del país y con ello se podía someter a la herramienta a ensayos más variados ${ }^{1}$. En segundo término, se valoró positivamente el tener un mejor conocimiento geográfico la comunidad autónoma, circunstancia que no es trivial a la hora de contrastar fehacientemente los resultados obtenidos.

\section{Desarrollo del trabajo}

\subsection{Tratamiento de la información. Descifrando el código SIOSE}

Como quedó señalado anteriormente, el modelo propuesto por el IGN posee el mérito de concentrar una cantidad de información ingente en un código cuya longitud tiene, por término medio, unas pocas decenas de caracteres. Con ello se consigue, por un lado, facilitar la distribución a los usuarios pero, por otra parte, tiene el coste de aumentar la dificultad que entraña explotar la fuente directamente, puesto que cualquier intento de utilizarla en detalle multiplica rápidamente la cantidad de datos a manejar.

La propuesta que se desarrolla con la herramienta de descifrado del SIOSE permite tabular los valores derivados del código, presentarlos en texto legible fácilmente y con una organización que posibilita manipular la información sin complicación excesiva. Partiendo de la estructura básica del código (porcentaje, etiqueta y atributos), se obtienen 33 campos diferentes con la información

\footnotetext{
${ }^{1}$ El mayor grado de complejidad radica en el hecho de haber más o menos niveles jerárquicos necesarios para describir los usos del suelo que componen un polígono en el SIOSE. En el caso de Asturias se ha llegado a alcanzar un cuarto umbral, mientras que, por ejemplo, en Cantabria o Castilla y León no se ha superado el tercero.
} 
Fernández Noguerol, S. (2017): "Desarrollo de herramientas para el tratamiento de la información y el análisis con SIG de los usos del suelo utilizando el SIOSE. Una aproximación al caso de Asturias.”, GeoFocus (Artículos), $n^{\circ} 20, p .233-$ 251. ISSN: $1578-5157$ http://dx.doi.org/10.21138/GF.573

contenida en la documentación técnica del IGN que permite análisis individuales o combinados de cada uno de ellos.

Para explicar mejor el proceso llevado a cabo, se tomará a modo de ejemplo el código de un polígono lo suficientemente complejo como para permitir exponer la estructura de datos de la tabla resultante y comentar las posibilidades de análisis espacial o estadístico que ofrece. Dentro de los datos que componen los usos del suelo asturianos en 2011, existe un caso en el que se llega al cuarto nivel jerárquico de coberturas para describir la ocupación de un espacio. Su ubicación está próxima al parque de carbones de la central térmica de Lada y a las afueras de la localidad de La Felguera, perteneciente al municipio de Langreo, que se sitúa en la cuenca del río Nalón. El código SIOSE del polígono ${ }^{2}$ es el siguiente:

I(50A(80UDS(80EDFem_20VAP)_20AAR(90PRDsc_10EDFva))_50IAS(60EDFnv_30ZAU_10VAP))

Analizando brevemente el ejemplo anterior, al comienzo se hace referencia a un mosaico irregular (nivel jerárquico 1) compuesto al $50 \%$ por "asociación" e "industrial aislada" (nivel jerárquico 2). La primera tiene un $80 \%$ de "urbanizado discontinuo" y un $20 \%$ de "asentamiento agrícola residencial" (nivel jerárquico 3), descomponiéndose ambas a su vez en otras coberturas de nivel jerárquico 4. Sin terminar de ver el código en su extensión, queda claro que la complejidad es considerable y su comprensión hace necesario revisar constantemente el documento "Descripción del modelo de datos y rótulo SIOSE v1.1” (IGN, 2015).

Frente a lo anterior, la tabla de atributos derivada de la ejecución del descifrador tiene como campos principales los mostrados en la tabla 1 . La ventaja es que se presentan al usuario todos los datos derivados del modelo definido por el IGN de forma organizada y clara, lo que permite en última instancia, un aprovechamiento muy detallado de una fuente cartográfica con grandes posibilidades de análisis. Por cada código SIOSE procesado, se inserta en la tabla tantas filas como coberturas componen el rótulo, de esta manera, recuperando el ejemplo seleccionado anteriormente, se añadirían doce registros. En total existen 33 campos, obviamente no todos tienen la misma importancia o potencial, y otros presentan la misma información en formato código o texto. A continuación, se explica la organización lógica y se muestran algunos ejemplos de los valores que pueden contener:

a) Campos relativos al polígono: son los cuatro primeros y contienen el identificador único del polígono, el código SIOSE y la superficie.

b) Campos referidos a la cobertura: son los 13 siguientes y representan los de mayor interés. Entre ellos destacan ordenados por el nivel de detalle en la desagregación de usos del suelo: tema, subtema, subtema de infraestructuras y nombre. A cada uno de ellos les acompaña el código o etiqueta de equivalencia.

c) Campos relacionados con los atributos: son los ocho últimos, divididos en dos grupos de cuatro cada uno. Esto se debe a que, si bien hay coberturas que no tienen atributos, es frecuente que tengan solo uno, siendo menos comunes las que alcanzan el máximo de dos.

\footnotetext{
${ }^{2}$ El código de identificación único (ID) del polígono es el: “2a01fa82-0cfe-45c2-8aed-a875212a3697”.
} 
Fernández Noguerol, S. (2017): "Desarrollo de herramientas para el tratamiento de la información y el análisis con SIG de los usos del suelo utilizando el SIOSE. Una aproximación al caso de Asturias.”, GeoFocus (Artículos), $n^{\circ} 20, p .233-$ 251. ISSN: $1578-5157$ http://dx.doi.org/10.21138/GF.573

Tabla 1. Relación de los campos principales del conjunto que componen la tabla obtenida por el descifrador del SIOSE.

\begin{tabular}{|c|c|c|}
\hline Nombre & $\begin{array}{c}\text { Tipo de } \\
\text { dato }\end{array}$ & Descripción \\
\hline Polígono OID & Entero & ID del polígono SIOSE en la tabla original \\
\hline Polígono ID & Texto & Identificador único del polígono SIOSE \\
\hline Polígono código SIOSE & Texto & Código del polígono SIOSE (rótulo) \\
\hline Polígono superficie & Doble & Superficie del polígono SIOSE \\
\hline Cobertura original & Texto & Código de la cobertura \\
\hline Cobertura posición en rótulo & Entero & Lugar que ocupa la cobertura en el rótulo \\
\hline Cobertura nivel jerárquico & Entero & $\begin{array}{l}\text { Nivel dentro del código SIOSE dónde se inscribe la } \\
\text { cobertura (valores de } 1 \text { a } 4 \text { ) }\end{array}$ \\
\hline Cobertura cobertura nodriza & Entero & $\begin{array}{l}\text { Indica cuál es la posición de nivel jerárquico anterior } \\
\text { en el que se inscribe (en base 0) }\end{array}$ \\
\hline Cobertura tipo & Texto & $\begin{array}{l}\text { Puede ser: simple, compuesta predefinida o compuesta } \\
\text { no predefinida (rel. comp. vid. IGN, 2015, pp. 14-17) }\end{array}$ \\
\hline Cobertura tema & Texto & $\begin{array}{l}\text { Por ejemplo: Arbolado forestal, cobertura artificial, } \\
\text { matorral... (vid. IGN, 2015, pp. 14-17) }\end{array}$ \\
\hline Cobertura subtema & Texto & $\begin{array}{l}\text { Por ejemplo: Aguas continentales, coníferas, industrial, } \\
\text { urbano mixto... (vid. IGN, 2015, pp. 14-17) }\end{array}$ \\
\hline Cobertura subtema infraestructuras & Texto & $\begin{array}{l}\text { Puede ser: energía, residuos, transporte, suministro de } \\
\text { agua y telecomunicaciones (vid. IGN, 2015, p. 16) }\end{array}$ \\
\hline Cobertura etiqueta & Texto & Abreviatura del nombre largo de la cobertura \\
\hline Cobertura nombre & Texto & Nombre de la cobertura (vid. IGN, 2015, pp. 14-16) \\
\hline Cobertura porcentaje original & Flotante & $\begin{array}{l}\text { Porcentaje de ocupación de la cobertura en el polígono } \\
\text { SIOSE en el que se inscribe }\end{array}$ \\
\hline Cobertura porcentaje derivado & Flotante & $\begin{array}{l}\text { Porcentaje de ocupación de la cobertura que solo } \\
\text { difiere del original en los niveles jerárquicos } 3 \text { y } 4\end{array}$ \\
\hline Cobertura superficie & Doble & $\begin{array}{l}\text { Se recalcula desde la del polígono en función al } \\
\text { porcentaje de ocupación original (niveles jerárquicos } 1 \\
\text { y } 2 \text { ), o el derivado (niveles jerárquicos } 3 \text { y } 4 \text { ) }\end{array}$ \\
\hline Atributo tipo & Texto & Siempre es "atributo" \\
\hline Atributo tema & Texto & $\begin{array}{l}\text { Por ejemplo: abancalado, cortas, es forzado, } \\
\text { plantación... (vid. IGN, 2015, pp. 14-17) }\end{array}$ \\
\hline Atributo etiqueta & Texto & Abreviatura del nombre completo del atributo \\
\hline Atributo nombre & Texto & Nombre del atributo (vid. IGN, 2015, p. 17) \\
\hline
\end{tabular}

Fte. Elaboración propia. 
Fernández Noguerol, S. (2017): "Desarrollo de herramientas para el tratamiento de la información y el análisis con SIG de los usos del suelo utilizando el SIOSE. Una aproximación al caso de Asturias.”, GeoFocus (Artículos), $n^{\circ} 20, p .233-$ 251. ISSN: $1578-5157$ http://dx.doi.org/10.21138/GF.573

En el caso de los datos del SIOSE de Asturias de 2005 hay 69549 polígonos que, tras ejecutar la herramienta de descifrado se convierten en 277125 coberturas, número que equivale a los registros guardados en la tabla final. Las cifras son similares para el 2011, dónde se pasa de 71067 a 284488 . Con el resultado obtenido se facilita la explotación analítica de la información desde dos aproximaciones complementarias: en primer término, se puede realizar cartografía temática $\mathrm{y}$, en segundo lugar, se permite profundizar en el conocimiento geográfico mediante el desarrollo de análisis espaciales más complejos.

4.2. Desarrollo de herramientas de análisis geográfico. El ejemplo de la perspectiva paisajística

La cantidad ingente de información que contiene el SIOSE hace que, con el mero hecho de confeccionar cartografía sobre los usos del suelo, se pueda comprender la configuración territorial de un espacio dado desde este punto de vista. Las posibilidades de estudio crecen también de forma considerable cuando los datos descifrados son tratados con las herramientas de análisis espacial que incluyen los SIG. De este modo y continuando con las decisiones adoptadas anteriormente, se ha implementado un script en ArcGIS que, utilizando el lenguaje nativo de geoprocesamiento de la plataforma "ArcPy", busca obtener una noción general del tipo de paisajes que se pueden contemplar desde las capitales de los municipios, tomando como ejemplo las de Asturias.

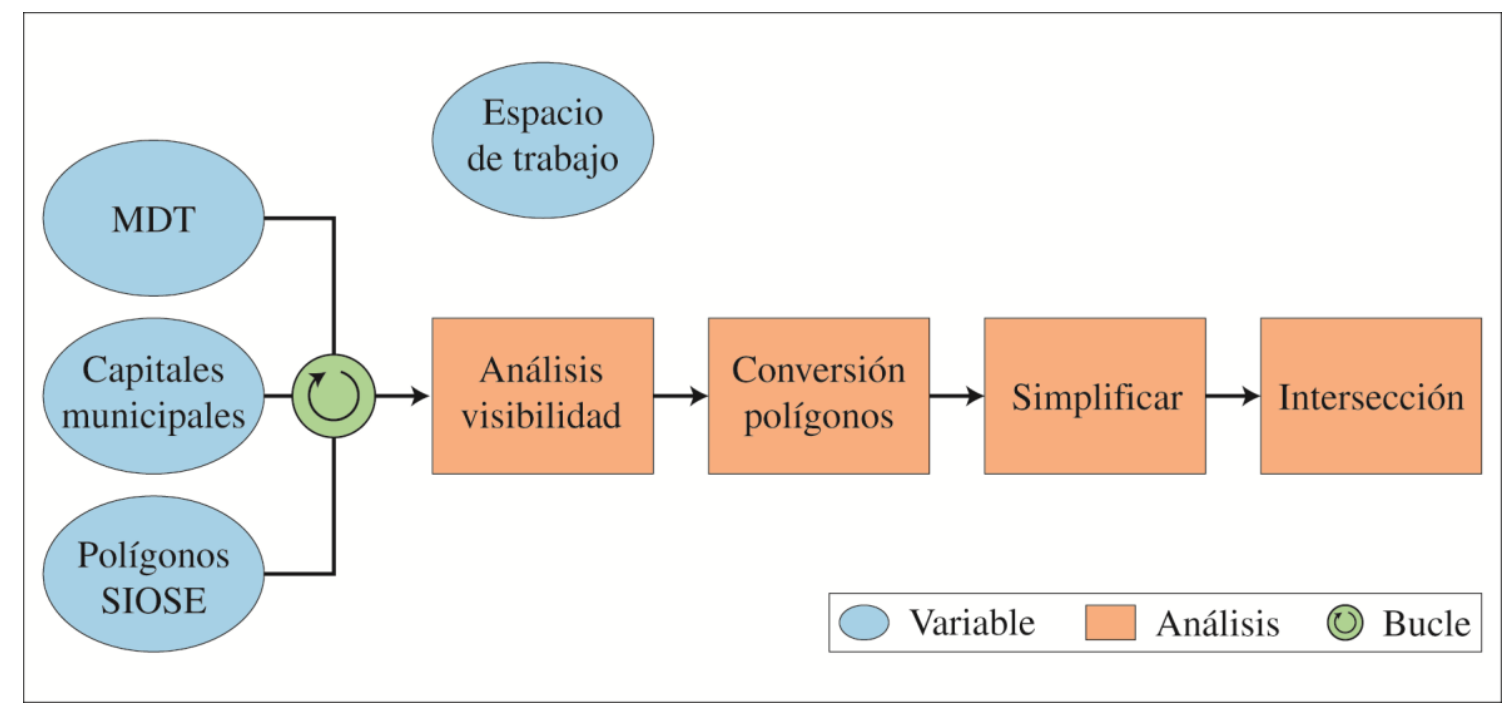

Figura 2. Diagrama de síntesis con la organización del código fuente de la herramienta para el análisis de la perspectiva paisajística.

Fte. Elaboración propia.

Como puede verse en la figura 2 , se necesitan cuatro parámetros o variables para ejecutar el estudio. El primero, denominado "espacio de trabajo", tiene por objeto guardar la ruta del disco duro dónde se almacenarán los resultados intermedios de carácter temporal y los definitivos, siendo lo ideal que se corresponda con una base de datos geográfica. Junto con el anterior, de carácter accesorio en el sentido analítico, lo realmente importante para alcanzar el objetivo es disponer de un Modelo Digital del Terreno (MDT), una clase de entidad de puntos donde se representen las 
Fernández Noguerol, S. (2017): "Desarrollo de herramientas para el tratamiento de la información y el análisis con SIG de los usos del suelo utilizando el SIOSE. Una aproximación al caso de Asturias.”, GeoFocus (Artículos), $n^{\circ} 20, p .233-$ 251. ISSN: $1578-5157$ http://dx.doi.org/10.21138/GF.573

capitales $^{3}$ y otra con geometría de polígonos con los usos del suelo del SIOSE, empleándose en este caso la obtenida con el descifrador.

Con todo ello se realizarán tantos análisis de perspectiva paisajística como localidades haya en la variable correspondiente, empleando para ello un bucle. Una vez iniciado el proceso, la primera herramienta de ArcGIS utilizada es la de "Visibilidad", cuyo fin es obtener los lugares que pueden contemplarse desde el punto de referencia ${ }^{4}$. Como el resultado intermedio obtenido en este punto es de tipo ráster, se hace una conversión a polígonos y, a continuación, éstos son sometidos a una simplificación para que eliminar los más pequeños o aquellos cuya forma es reminiscente de la trama de celdas. El último paso llevado a cabo por el script es una intersección entre los polígonos obtenidos con el análisis de cuencas visuales y las coberturas descifradas del SIOSE, con ello se obtienen los usos existentes en cada lugar visible desde el lugar de observación.

Al mismo tiempo que se ejecutan las tareas principales del análisis, se realizan otras de carácter secundario que facilitan el tratamiento posterior de la información. Por ejemplo, tomando los datos de la tabla de atributos de las capitales municipales, se realiza una sustitución de los nombres de los nuevos ráster o clases de entidad para evitar la presencia de caracteres latinos que puedan generar problemas de funcionamiento en ArcGIS 5 . También se incorporan tres nuevos campos al resultado de la intersección, cuyo fin es disponer del código municipal, el nombre del municipio y el de su capital.

Tras la ejecución del script se conserva la superposición de coberturas que caracterizaba a los datos descifrados del SIOSE, pero solo se mantienen las ocupaciones de los lugares visibles desde la localidad de referencia. El resultado se fundamenta en la decisión de mantener disponible la mayor cantidad de datos en todas las fases de tratamiento de la información para que, llegado el caso, el usuario tenga múltiples opciones de elección y pueda cribar aquellos aspectos que le resulten más convenientes en cada momento. En esencia se sigue la misma filosofía que en la fuente original del IGN, pero los datos se presentan de una forma más accesible, que es el objetivo pretendido en este trabajo.

En el ejemplo de la perspectiva paisajística se optó por mantener exclusivamente el uso mayoritario de entre aquellos que conformaban el polígono original. Los más sencillos de obtener fueron los que tenían por tipo "Cobertura compuesta predefinida". Seguidamente, se cribaron los que están definidos como "Cobertura compuesta no predeterminada", cuyo procedimiento fue más complejo por la amplia variabilidad de situaciones presentes, que requirieron realizar diferentes combinaciones de los campos "Cobertura tipo" y "Cobertura posición en rótulo". En el tercer y último paso se eliminaron los polígonos de "Mares y océanos" por no representar usos del suelo en sentido estricto. El proceso de selección se hizo mediante sentencias SQL, imprescindibles para el análisis de la información almacenada en bases de datos.

Aunque la metodología desarrollada para conseguir este último procedimiento no ha sido programada, los pasos que el usuario debe seguir para alcanzar la simplificación podrían ser automatizados. Las alternativas son varias: mediante un script de ArcPy o durante la ejecución de la herramienta de descifrado. En el segundo caso sería más práctico, porque solo habría que incorporar

\footnotetext{
${ }^{3}$ La fuente utilizada ha sido el "Nomenclátor geográfico de municipios y entidades de población", distribuido por el IGN a través del Centro de descargas del Centro Nacional de Información Geográfica (CNIG).

${ }^{4}$ El MDT usado tiene un paso de malla de 5 metros (PNOA-IGN). La altura de observación fue de un metro.

${ }^{5}$ Tildes, apóstrofes, eñes, puntos, espacios, etc.
} 
Fernández Noguerol, S. (2017): "Desarrollo de herramientas para el tratamiento de la información y el análisis con SIG de los usos del suelo utilizando el SIOSE. Una aproximación al caso de Asturias.”, GeoFocus (Artículos), $n^{\circ} 20, p .233-$ 251. ISSN: $1578-5157$ http://dx.doi.org/10.21138/GF.573

un campo nuevo de tipo numérico a la tabla de atributos que, partiendo de 1 y con incrementos unitarios, guardase el orden de mayor a menor de los porcentajes derivados de las coberturas que integran un polígono.

\section{Resultados}

\subsection{El código SIOSE decodificado}

Para poder trabajar fácilmente con el descifrador del SIOSE se ha creado un "Add-in" compatible con "ArcMap 10.5". Gráficamente, se presenta como una caja de herramientas cuya apariencia es muy similar a la de cualquier otra realizada por la empresa ESRI. Con ello se separa la implementación lógica del código fuente descrito en el apartado 4.1, de la interfaz de usuario para que este no esté obligado a adquirir conocimientos adicionales para poder trabajar con la utilidad. Los datos pueden ser trabajados de nueve maneras distintas, lo que representa una variedad de opciones considerable que ofrece amplias posibilidades de procesamiento e interacción entre distintos entornos de trabajo, facilitando el tratamiento de los datos. Como puede verse en la figura 3, los contenidos están organizados mediante dos menús (datos y selección) que agrupan varias funciones afines entre sí. Además, hay tres botones individuales que brindan utilidades complementarias de análisis sobre elementos concretos.

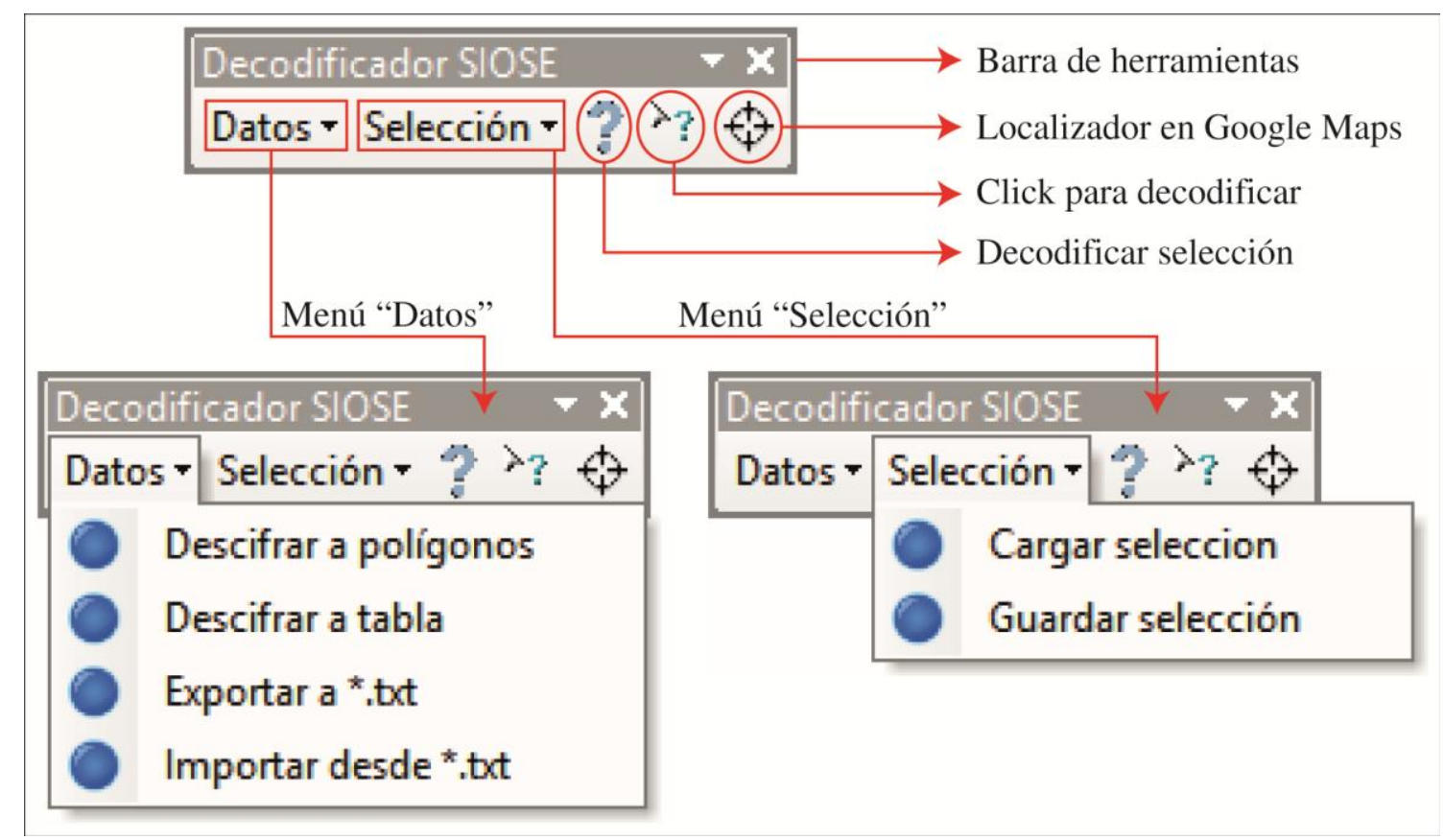

Figura 3. Diagrama de la barra de herramientas del "Decodificador SIOSE" para ArcGIS que muestra las funciones de los menús y los botones.

Fte. Elaboración propia.

El menú de datos es el principal y tiene como objetivo fundamental decodificar el código SIOSE. Es importante destacar que, como paso previo, los datos originales deben ser importados 
Fernández Noguerol, S. (2017): "Desarrollo de herramientas para el tratamiento de la información y el análisis con SIG de los usos del suelo utilizando el SIOSE. Una aproximación al caso de Asturias.”, GeoFocus (Artículos), $n^{\circ} 20, p .233-$ 251. ISSN: $1578-5157$ http://dx.doi.org/10.21138/GF.573

por el usuario a una base de datos geográfica y, posteriormente, cargados a la vista de ArcMap. Con la capa correspondiente seleccionada en la tabla de contenidos, las dos primeras opciones permiten descifrar la información de base en bloque, si bien la primera crea una clase de entidad nueva que conserva la representación gráfica, mientras que la segunda genera exclusivamente una tabla de atributos. La exportación e importación de esta última a un archivo de texto separado por comas facilita la interoperabilidad con otros entornos de trabajo estadístico como "Microsoft Excel" o "RStudio", entre otros.

Una vez se han decodificado los datos del SIOSE y se realizan diferentes selecciones sobre temas concretos o de cualquier otra índole, resulta útil poder guardar las referencias de los elementos que cumplen con los criterios definidos por el usuario, de esta manera pueden retomarse posteriormente para continuar con el trabajo. Esa es la función que cumple el menú "Selección", en primera instancia almacena los identificadores de los elementos destacados en un archivo guardado en el disco duro para, posteriormente, recuperar la selección. Como pueden salvaguardarse tantas como sean necesarias, la utilidad permite diferenciar unas de otras mediante un nombre y una descripción de los contenidos. Los valores se escriben en un archivo con extensión "*.sel" que internamente alberga una estructura de datos XML de elaboración propia.

Los botones "Decodificar selección" y "Click para decodificar" se ubican en el contexto general de la barra de herramientas, cumpliendo ambos la función presentar de forma legible las características de las coberturas que componen un polígono seleccionado. La diferencia entre ambas herramientas radica en que, en el primer caso, es necesario tener seleccionada una entidad en la tabla de atributos, mientras que, en la segunda, la entidad se elige directamente con un clic sobre cualquier polígono SIOSE que esté representado en la vista del mapa.

Como puede verse en la figura 4 , los resultados se presentan en una ventana nueva que emerge sobre la principal de ArcMap y que representa, en la mitad superior, las coberturas desglosadas jerárquicamente de acuerdo con su disposición en el código original. Recuperando el ejemplo mencionado en el apartado 4.1, la legibilidad es considerablemente mejor ahora y ello permite que el usuario pueda ver fácilmente cómo se organizan los distintos usos del suelo presentes en el polígono. De forma complementaria, en la parte inferior se añade la tabla ${ }^{6}$ con toda la información de cada cobertura para que puedan ser consultadas según convenga.

La ventana incorpora también una barra de herramientas con diversas opciones: expandir o contraer los nodos jerárquicos, mostrar en Google Maps el centroide del polígono y mostrar gráfico. Esta última es la más interesante desde el punto de vista del análisis espacial, ya que permite visualizar fácilmente la proporción que ocupa cada uso del suelo con respecto al total mediante un diagrama circular, cuya representación se genera automáticamente, del mismo modo que el título y la leyenda. A través del menú de opciones, se puede exportar el resultado como imagen de tipo "png" o como archivo vectorial/ráster "emf" para, por ejemplo, editarlo en "Adobe Photoshop" o "Illustrator" con vistas a incorporarlo a informes técnicos, publicaciones científicas, páginas web, etc.

\footnotetext{
${ }^{6}$ La estructura de campos es la mostrada en la tabla 1.
} 
Fernández Noguerol, S. (2017): "Desarrollo de herramientas para el tratamiento de la información y el análisis con SIG de los usos del suelo utilizando el SIOSE. Una aproximación al caso de Asturias.”, GeoFocus (Artículos), $n^{\circ} 20, p .233-$ 251. ISSN: $1578-5157$ http://dx.doi.org/10.21138/GF.573

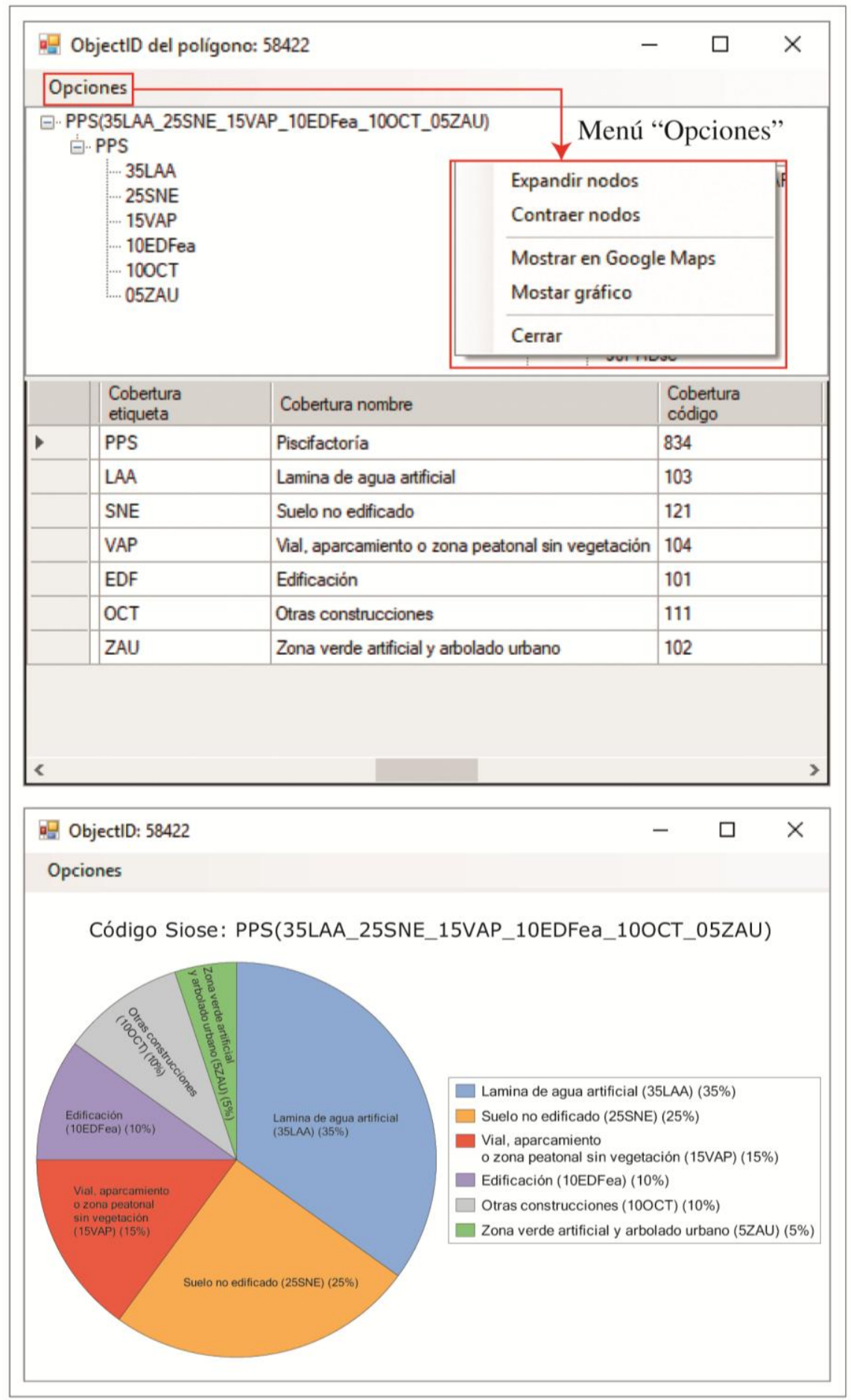

Figura 4. Captura de la ventana con el código desglosado (arriba) y de la que muestra el gráfico circular con las coberturas de un polígono (abajo).

Fte. Elaboración propia. 
Fernández Noguerol, S. (2017): "Desarrollo de herramientas para el tratamiento de la información y el análisis con SIG de los usos del suelo utilizando el SIOSE. Una aproximación al caso de Asturias.”, GeoFocus (Artículos), $n^{\circ} 20, p .233-$ 251. ISSN: $1578-5157$ http://dx.doi.org/10.21138/GF.573

Complementariamente con las utilidades anteriores, el último botón de la barra de herramientas realiza la función de localizar en el visor de fotografía aérea de Google Mapas el punto marcado mediante un clic del ratón en la vista del mapa de ArcMap. De esta manera se puede hacer un reconocimiento virtual del área de estudio para comprender mejor cuáles son sus características. Esto resulta especialmente útil cuando no se dispone de ortofotografías o si se desea incorporar en un informe una referencia al emplazamiento del polígono SIOSE.

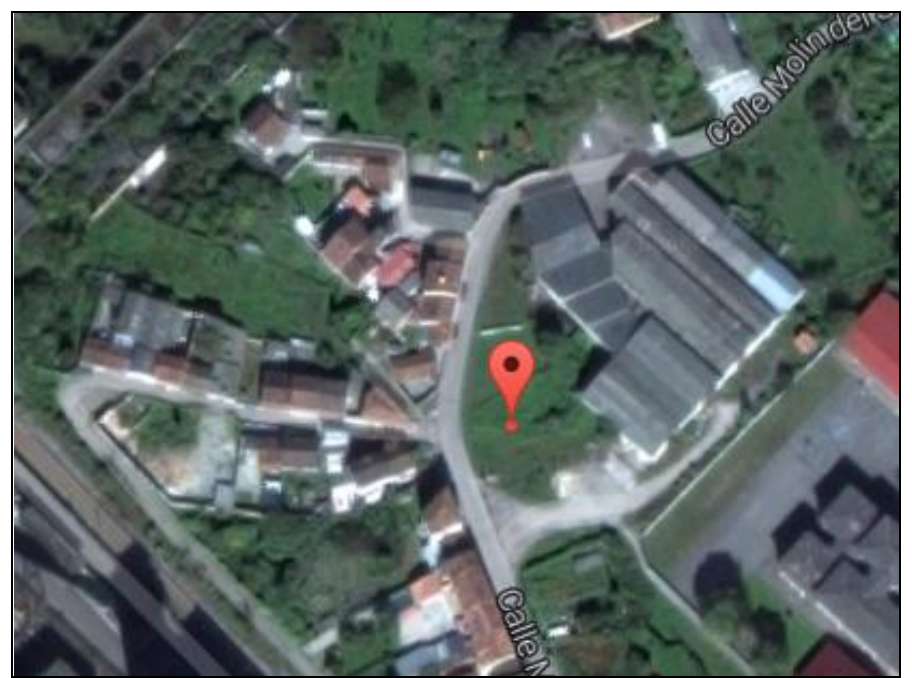

Figura 5. Captura de pantalla mostrando en Google Maps el punto marcado en ArcMap. Fte. Google Maps. Elaboración propia.

5.2. Breve aproximación a los usos del suelo de Asturias y la perspectiva paisajística a nivel local

Una vez que el código de los polígonos del SIOSE es descifrado y se almacenan sus atributos en tablas, se abren unas posibilidades de análisis espacial muy amplias que permiten realizar estudios sobre la materia a nivel estatal, regional y local. Dejando al margen la escala nacional, cuya escala de referencia es más apropiada para otras fuentes como el Corine Land Cover, el nivel de detalle de los datos suministrados por el IGN es más apropiado para las provincias y, especialmente, para los municipios.

Antes de abordar la cartografía de detalle que se presenta en este epígrafe, es conveniente realizar una aproximación breve a la distribución general de las coberturas presentes en Asturias, de este modo el lector que no esté familiarizado con la región puede obtener una visión general del conjunto. Para ello, se ha optado por aplicar la simbología que se presenta en el Web Map Service (WMS) del SIOSE ya que, mediante la agrupación de los usos del suelo en dos niveles jerárquicos, se obtiene una visión simplificada que facilita la lectura. El primero de ellos está pensado para escalas inferiores al 1:150 000 y cuenta con 10 elementos, lo que facilita las representaciones regionales. El segundo, para escalas mayores a la anterior, amplía notablemente el detalle en el ámbito subregional y representa 22 tipologías, siendo fundamentalmente los espacios antrópicos los beneficiados en el incremento de clases. Aunque aquí no se han representado, en lo local se emplearía el conjunto completo de tipologías a escala 1:25 000 . 


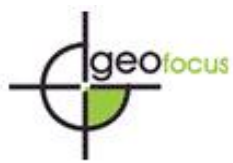

Fernández Noguerol, S. (2017): "Desarrollo de herramientas para el tratamiento de la información y el análisis con SIG de los usos del suelo utilizando el SIOSE. Una aproximación al caso de Asturias.”, GeoFocus (Artículos), $n^{\circ} 20, p .233-$ 251. ISSN: $1578-5157$ http://dx.doi.org/10.21138/GF.573

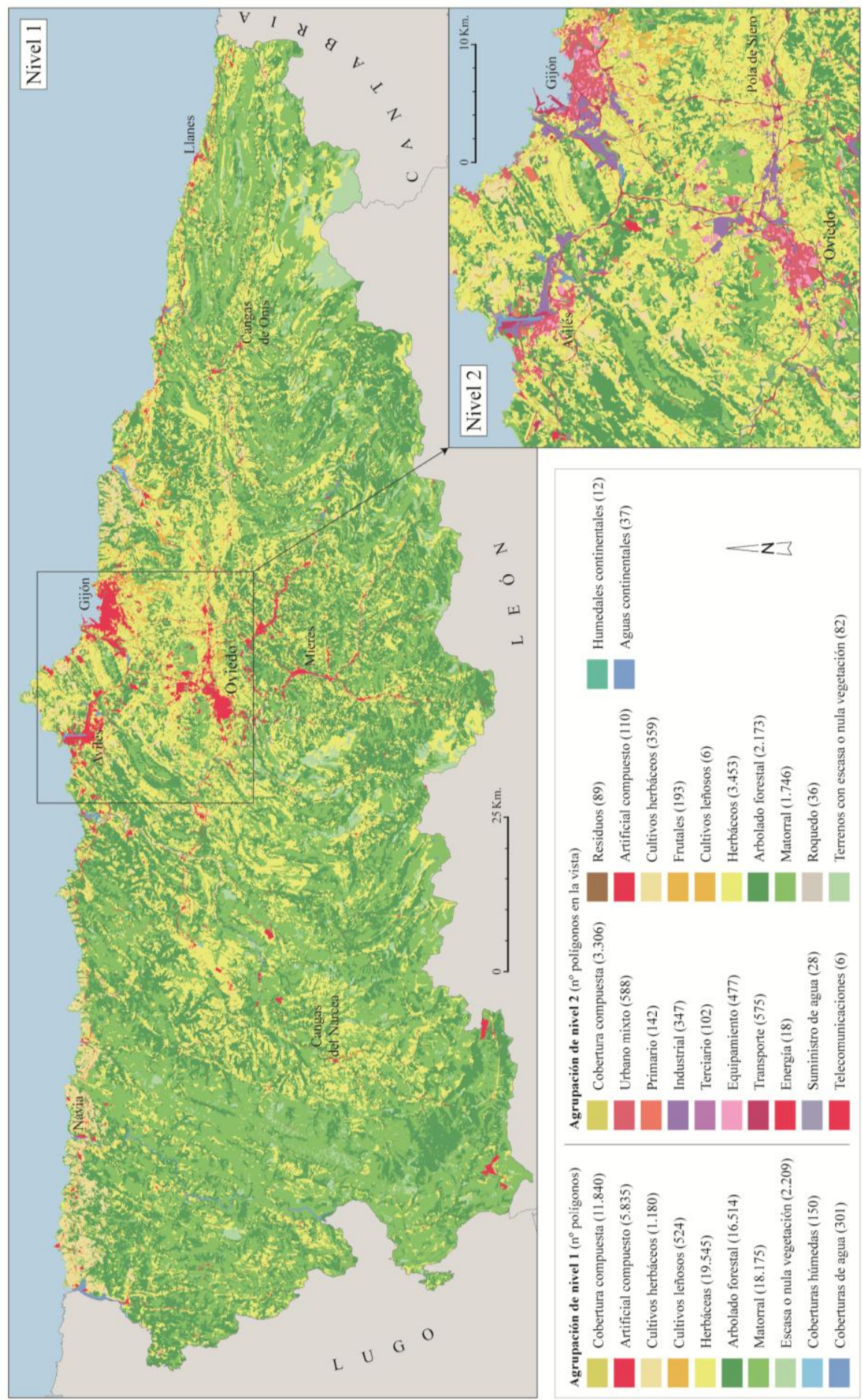

Figura 6. Usos del suelo predominantes en Asturias agrupados según los niveles 1 y 2 definidos por el IGN.

Fte. SIOSE de Asturias con fecha de referencia de 2011, Instituto Geográfico Nacional. Elaboración propia 
Fernández Noguerol, S. (2017): "Desarrollo de herramientas para el tratamiento de la información y el análisis con SIG de los usos del suelo utilizando el SIOSE. Una aproximación al caso de Asturias.”, GeoFocus (Artículos), $n^{\circ} 20, p .233-$ 251. ISSN: $1578-5157$ http://dx.doi.org/10.21138/GF.573

Según queda cartografiado en la figura 6, el matorral es la cobertura con mayor extensión en Asturias $\left(\sim 10600 \mathrm{~km}^{2}\right)$, con casi $3500 \mathrm{~km}^{2}$ repartidos en más de 16500 polígonos. Muestra una distribución generalizada y bastante homogénea, si bien destaca la concentración en torno a la franja más occidental de la región y, en el sentido contrario, su menor presencia en los espacios llanos del centro de la provincia. Por tanto, este uso del suelo está vinculado a los espacios montañosos, donde suele asociarse a otros como el arbolado forestal y las herbáceas. El primero, con poco más de 15100 polígonos, supera los $3200 \mathrm{~km}^{2}$ y está confinado a las montañas de la divisoria con León en la mayoría de los casos, aunque también genera manchas significativas en las proximidades del litoral. Las segundas, que superan los $2600 \mathrm{~km}^{2}$, tienen la mayor cantidad de polígonos (18 421) porque incluyen los prados, cuya compartimentación es muy importante a causa del tipo de propiedad minifundista que predomina en el norte de España.

La distribución de las coberturas predominantes responde a la intrincada orografía montañosa a la que se adapta, no sin dificultad, la actividad ganadera que domina el sector agrario asturiano. En el extremo opuesto se hayan los espacios urbanizados que se asientan sobre los terrenos llanos y que alcanzan el mayor grado de desarrollo en el surco prelitoral sobre el que ha crecido la capital de la Comunidad Autónoma, aunque tampoco puede pasar por alto la franja costera.

Obviando ahora las tres coberturas señaladas hasta el momento, para contemplar los espacios urbanos del centro de Asturias representados según la agrupación de usos de nivel 2, destaca la extensión de tres localidades: Oviedo, Gijón y Avilés, a las que acompañan otras de menor rango cómo Pola de Siero. El centro de estas ciudades está compuesto por edificación residencial de alta densidad, mientras que en los espacios de borde, de construcción más reciente, se intercalan distintos equipamientos que oxigenan la trama urbana. En la periferia de Gijón y Avilés, predominan los usos industriales históricos, mientras que en la capital provincial destacan polígonos empresariales como los de Silvota, Asipo, Colloto o Meres, y grandes superficies comerciales como "Intu Asturias" o "Azabache". El conjunto queda conectado por las vías de comunicación de alta capacidad que, con su morfología estrecha y alargada, compartimentan el territorio notablemente.

Antes de terminar con la visión de conjunto, conviene hacer mención especial a la categoría "Cobertura compuesta" que, en el caso de Asturias, se corresponde en un 96,43\% de los casos con coberturas de tipo "Asentamiento agrícola residencial", es decir, poblamiento rural de carácter disperso. A pesar de su atomización (3 188 polígonos), la importancia de las aldeas y los pueblos es tal en el territorio que, solo teniendo en consideración los ejemplos presentes en el centro de la región, se rozan los $108 \mathrm{~km}^{2}$ de extensión, superando cómodamente los 4,4 km² de las grandes ciudades (570 polígonos).

A nivel local es dónde verdaderamente queda de manifiesto la potencialidad del SIOSE ya que el gran detalle de la fuente permite superar las limitaciones que impone el Corine Land Cover. Llegados a este punto se añade un nivel mayor de complejidad al análisis, que se fundamenta en la combinación de los datos de usos del suelo, con modelos digitales del terreno y análisis de la visibilidad. El enfoque, denominado "perspectiva paisajística", permite obtener una noción general del tipo de paisaje que rodea a un espacio dado, habiéndose elegido en este caso las 78 capitales municipales de Asturias. Puesto que la intención de este artículo no es la de representar exhaustivamente los resultados obtenidos para cada concejo, se ha optado por cartografiar y comentar cuatro ejemplos significativos. 
Fernández Noguerol, S. (2017): "Desarrollo de herramientas para el tratamiento de la información y el análisis con SIG de los usos del suelo utilizando el SIOSE. Una aproximación al caso de Asturias.”, GeoFocus (Artículos), $n^{\circ} 20, p .233-$ 251. ISSN: $1578-5157$ http://dx.doi.org/10.21138/GF.573

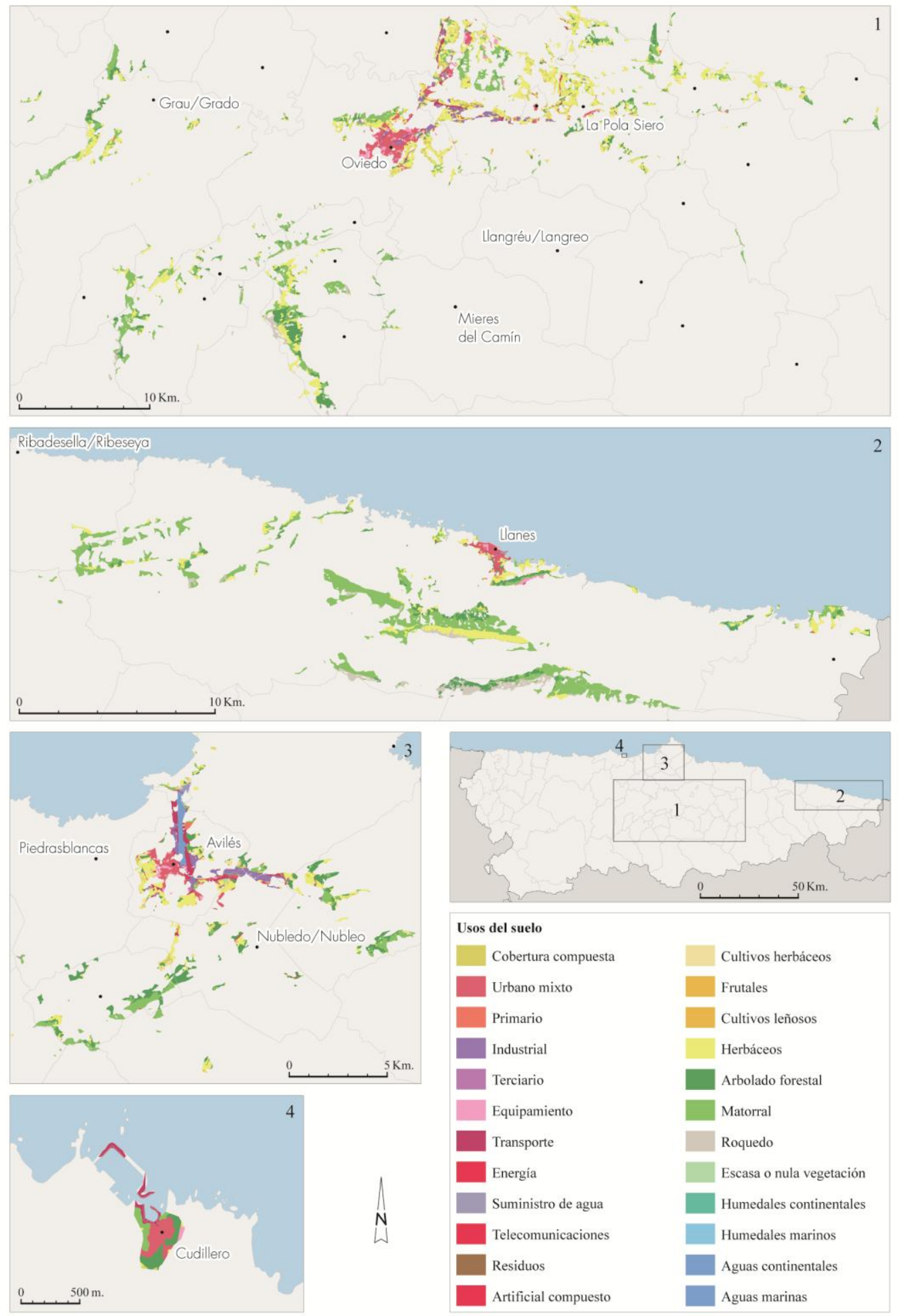

Figura 7. Perspectiva paisajística de Oviedo, Llanes, Avilés y Cudillero.

Fte. SIOSE de Asturias con fecha de referencia de 2011, Instituto Geográfico Nacional. Elaboración propia. 
Fernández Noguerol, S. (2017): "Desarrollo de herramientas para el tratamiento de la información y el análisis con SIG de los usos del suelo utilizando el SIOSE. Una aproximación al caso de Asturias.”, GeoFocus (Artículos), $n^{\circ} 20, p .233-$ 251. ISSN: $1578-5157$ http://dx.doi.org/10.21138/GF.573

Como queda de manifiesto en la figura 7, los análisis realizados para Oviedo, Llanes, Avilés y Cudillero muestran diferencias notables entre sí. La principal es la importancia que tiene el emplazamiento, puesto que de él depende que haya más o menos superficie de terreno visible y que, por consiguiente, se abarque mayor o menor variedad de usos del suelo. En segundo lugar, la especialización económica en uno u otro sector confiere características específicas a las coberturas que rodean cada localidad lo que, en última instancia, define el paisaje que la circunda. Los ejemplos elegidos permiten mostrar con claridad los conceptos señalados.

Oviedo es una de las ciudades con más campo visual de la región por situarse sobre un promontorio ubicado en las proximidades del centro geográfico de Asturias. Salvo por las estribaciones del Monte Naranco que ejercen de barrera infranqueable por el Noroeste, desde la capital provincial se puede ver la mitad oriental del surco prelitoral hasta Pola de Siero, la Sierra del Aramo al Suroeste y las sierras de Pedrorio y Miranda al Oeste de Grado, sumando en total 120 $\mathrm{km}^{2}$. Las coberturas que predominan en la aureola de los primeros cinco kilómetros con respecto al punto de referencia son las de tipo urbano, con más de $6 \mathrm{~km}^{2}$, a las que supera el poblamiento residencial de morfología dispersa (7,3). A continuación están los usos industriales $(2,7)$, las infraestructuras de transporte $(2,5)$ y los equipamientos $(2,4)$. En el resto del espacio divisado, lo urbano cede el protagonismo a las herbáceas que suman $44 \mathrm{~km}^{2}$, el matorral (35) y el arbolado forestal (14).

En el caso de Llanes la visibilidad se ve limitada al Norte por las aguas del Mar Cantábrico y al Sur por la muralla que supone la Sierra de Cuera, lo que ciñe el resultado del análisis a una franja estrecha y alargada de $31 \mathrm{~km}^{2}$ con disposición E-W. El uso urbano mixto es el predominante en la villa (90 Has.), pero este es muy limitado en superficie si es comparado con el matorral que coloniza la mayor parte de los espacios de montaña circundantes $\left(17,6 \mathrm{~km}^{2}\right)$, seguido de las herbáceas $(5,7)$, el arbolado forestal $(3,4)$ o el roquedo de las cumbres de los Picos de Europa $(2,7)$. La Autovía del Cantábrico no es visible desde el casco histórico, por lo que, salvo la construcción residencial, el paisaje natural es mayoritario.

Aun siendo una localidad litoral, Avilés se sitúa a unos cuatro kilómetros tierra a dentro de la costa del Cantábrico, a la que accede a través de la ría a la que da nombre y que ocupa una superficie de unas 105 hectáreas. En los $33 \mathrm{~km}^{2}$ que se pueden observar desde la ciudad, la industria tiene una importancia superlativa con 172 Has, una docena más que los usos netamente urbanos. Tampoco es baladí que las infraestructuras de transportes sumen 150 Has., puesto que como los anteriores se concentran en un área a menos de cinco kilómetros del punto de referencia. Por encima de esa distancia, el matorral, las herbáceas y los espacios forestales ocupan los montes del tercio meridional.

Por último, en Cudillero se evidencia con claridad meridiana hasta qué punto el emplazamiento limita las posibilidades de contemplar el paisaje. Situada bajo el nivel topográfico de la rasa costera en un acantilado retranqueado con forma de anfiteatro, es la capital municipal con menor visibilidad de Asturias al no superar las 16 Has. Como se ve en el mapa, los usos urbanos representan la mayor proporción de la villa (35\%), seguidos de las infraestructuras de transporte que están integradas exclusivamente por los diques de abrigo del puerto pesquero ( 20\%). Justo en el límite inferior de la llanura litoral aparecen una exigua representación de 4 hectáreas de herbáceas y poco más de dos de matorral. 
Fernández Noguerol, S. (2017): "Desarrollo de herramientas para el tratamiento de la información y el análisis con SIG de los usos del suelo utilizando el SIOSE. Una aproximación al caso de Asturias.”, GeoFocus (Artículos), $n^{\circ} 20, p .233-$ 251. ISSN: $1578-5157$ http://dx.doi.org/10.21138/GF.573

\section{Conclusiones}

En este artículo se pretendía desarrollar una herramienta que automatizase la decodificación del código del Sistema de Información sobre Ocupación del Suelo de España y que facilitase el acceso al gran nivel de detalle que tiene esta fuente fundamental para el estudio de los usos del país. Complementariamente se buscaba explotar los resultados obtenidos mediante la confección de cartografía temática y valorar, posteriormente, las posibilidades de profundizar en el conocimiento geográfico mediante el desarrollo de nuevas herramientas de análisis espacial, tomando como objeto de estudio la provincia de Asturias.

En cuanto al primero de los objetivos, se ha conseguido crear una utilidad que facilita considerablemente el uso del SIOSE mediante la implementación de una herramienta informática que transforma los datos originales en una tabla de datos con una estructura clara, fácilmente legible y que evita la consulta constante de la documentación técnica del IGN. Gracias a ello, el usuario obtiene como principal ventaja práctica, una mayor facilidad para la elaboración de cartografía temática, puesto que no es necesario llevar a cabo operaciones de tratamiento de la información adicionales.

Por último, combinando los resultados de base con el potencial de los Sistemas de Información Geográfica, se pueden configurar modelos de análisis más elaborados que amplían las posibilidades de estudio y, por tanto, de comprensión de la realidad geográfica. El ejemplo planteado en el artículo bajo el nombre de "perspectiva paisajística" viene a corroborar la utilidad de la herramienta desarrollada ya que, gracias a la tabla de datos que obtiene, posibilita múltiples aplicaciones a nivel estatal, regional y local.

\section{Referencias bibliográficas}

Alcántara, J.; Romero, D.; Romero, A.; Ghishlanzoni, M.; Guerrero, J. J.; Giménez de Azcárate, F.; Cáceres, F. y Moreira, J. M. (2014): "Obtención de horizontes visuales a partir del Sistema de Visibilidad de Andalucía (REDIAM) y su aplicación en la identificación de unidades del paisaje", en Asociación de Geógrafos Españoles (AGE), Universidad de Alicante y Colegio de Geógrafos de España (Eds.): Tecnologías de la información para nuevas formas de ver el territorio: XVI Congreso Nacional de Tecnologías de Información Geográfica, celebrado del 25 al 27 de junio de 2014 (Alicante), Madrid, AGE, pp. 302-312.

Burriel Moreno, J. A.; Ibañez i Martí, J. J. y Masó Pau, J. (2008): “SIOSE en Cataluña: obtención a partir del mapa de cubiertas del suelo de Cataluña", en Hernández, L. y Parreño, J. M. (Eds.): Tecnologías de la Información Geográfica para el desarrollo territorial, Las Palmas de Gran Canaria, Servicio de publicaciones y difusión científica de la ULPGC, pp. 876-889.

Cantarino Marí, I. (2013): "Elaboración y validación de un modelo jerárquico derivado de SIOSE”, Revista de Teledetección, 39, pp. 5-21. [Consulta: 10-03-2017]. Disponible en: http://www.aet.org.es/?q=revista39-2

Del Bosque González, I.; Arozamena Villar, A.; Villa Alcázar, G.; Valcárcel Sanz, N. y Porcuna Fdez-Monasterio, A. (2005): "Creación de un sistema de información geográfico de ocupación del suelo en España. Proyecto SIOSE", en Arbelo, M.; González, A. y Pérez, J. C. (Eds.): Teledetección. Avances en la observación de la Tierra, Puerto de la Cruz, Universidad de la Laguna, pp. 255-262. 
Fernández Noguerol, S. (2017): "Desarrollo de herramientas para el tratamiento de la información y el análisis con SIG de los usos del suelo utilizando el SIOSE. Una aproximación al caso de Asturias.”, GeoFocus (Artículos), $n^{\circ} 20, p .233-$ 251. ISSN: $1578-5157$ http://dx.doi.org/10.21138/GF.573

Delgado Hernández, J. (2015): Generalización del SIOSE en la producción del CORINE Land Cover 2012 (Copernicus) con FME y ArcGIS. Instituto Geográfico Nacional. [Consulta: 10-032017]. Disponible en: http://www.fmewt.es/img/tour/ES\%202015/05_Generalizacion_SIOSEtoCLC_conFME.pdf

Díaz Manso, J. M.; Aller González, D.; Martín Rosón, A.; Barcia Noia, B. y Pereira Paula, S. (2005): "Dúas perspectivas sobre a cartografía de coberturas e usos do solo en Galicia", Revista Galega de Economía, vol. 16, núm. 1, pp. 71-94.

Fernández Noguerol, S. (2015): Transformaciones territoriales recientes en el valle del Caudal como consecuencia de la reconversión industrial. Oviedo, tesis doctoral inédita.

Goerlich Gisbert, F. (2013): “Áreas rurales y coberturas del suelo” en Fundación BBVA (Ed.): Documentos de trabajo, Bilbao, Fundación BBVA.

Goerlich Gisbert, F. J. y Cantarino Martí, I. (2013): Zonas de morfología urbana. Coberturas del suelo y demografía. Bilbao, Fundación BBVA.

Instituto Geográfico Nacional (2015): Descripción del modelo de datos y rótulo SIOSE, Madrid, Ministerio de Fomento. [Consulta: 16-01-2017]. Disponible en http://centrodedescargas.cnig.es

Instituto Geográfico Nacional (2017): Documentación, Madrid, Ministerio de Fomento. [Consulta: 10-03-2017]. Disponible en http://www.siose.es/web/guest/documentacion

Lasanta, T.; Errea Abad, Ma P.; Vicente-Serrano, S. M. y Arnáez, J. (2011): "La diversidad de la cubierta vegetal en campos abandonados del Leza y Jubera (Sistema Ibérico, La Rioja) a partir del SIOSE”, en Zubía. Monográfico. Revista de ciencias. Logroño, Instituto de Estudios Riojanos, 23, pp. 55-78.

Martí-Ciriquián, P.; Nolasco-Cirugeda, M. y Serrano-Estrada, L. (2014): "Centro urbanos vs sprawl: expansión urbana y equipamientos en las capitales valencianas", en Università degli Studio Roma Tre (Ed.): International Conference Virtual City and Territory. "9 ${ }^{\circ}$ Congresso Città e Territorio Virtuale, Roma, 2, 3 e 4 ottobre 2013, Roma, Universidad de Roma, pp. 398-408.

Moreira, J. M.; Quijada, J.; Ortega, E.; Romero, D. y Gil, Y. (2008): “El proyecto SIOSE en Andalucia", en Hernández, L. y Parreño, J. M. (Eds.): Tecnologías de la Información Geográfica para el desarrollo territorial, Las Palmas de Gran Canaria, Servicio de publicaciones y difusión científica de la ULPGC, pp. 941-953.

Pérez Hernández, H. (2015): Información geográfica de cambios en usos y coberturas del suelo: Montaña de Taco y sus aledaños (Buenavista del Norte y Los Silos, Tenerife, 1956-2013. Ensayo de adaptación del modelo SIOSE a escala 1:5.000 en el ámbito de la manzana cartográfica que incluye el topónimo de referencia, La Laguna (Tenerife), Universidad de la Laguna.

Rodríguez Gozalo, J. (2012): Evolución potencial de los usos del suelo en Asturias a través del análisis de variables territoriales. Mieres, Universidad de Oviedo.

Rosa Fernández, J. P. y Tudela Serrano, Mㄹ L. (2013): “Avance en la actualización de la evolución de los usos del suelo en el espacio periurbano "Huerta de Murcia" (Región de Murcia, España). Revisión SIOSE 2005-2009”, Papeles de Geografía, 57-58, pp. 225-241.

Rubio Díaz, J. A. y García Llamas, S. Ma (2008): "Intergraph - Control de calidad SIOSE con Geomedia”, en Hernández, L. y Parreño, J. M. (Eds.): Tecnologías de la Información Geográfica 
Fernández Noguerol, S. (2017): "Desarrollo de herramientas para el tratamiento de la información y el análisis con SIG de los usos del suelo utilizando el SIOSE. Una aproximación al caso de Asturias.”, GeoFocus (Artículos), $n^{\circ} 20, p .233-$ 251. ISSN: $1578-5157$ http://dx.doi.org/10.21138/GF.573

para el desarrollo territorial, Las Palmas de Gran Canaria, Servicio de publicaciones y difusión científica de la ULPGC, pp. 955-959.

Santos Preciado, J. M.; Prieto Flores, M. E.; Borderías Uribeondo, M. P.; Azcárate Luxán, M.V.; Cocero Matesanz, D.; Muguruza Cañas, M. C. y Santa Cecilia Mateos, F. (2014): "Análisis de la estructura urbana, mediante bases de datos de detalle. Aplicación al estudio del sector 3, barrio residencial del municipio de Getafe", en Asociación de Geógrafos Españoles (AGE), Universidad de Alicante y Colegio de Geógrafos de España (Eds.): Tecnologías de la información para nuevas formas de ver el territorio: XVI Congreso Nacional de Tecnologías de Información Geográfica, celebrado del 25 al 27 de junio de 2014 (Alicante), Madrid, AGE, pp. 663-675.

Valcarcel, N.; Villa, G.; Arozamena, A.; García-Asensio, L.; Caballero, M. E.; Porcuna, A.; Domenech, E. y Peces. J. J. (2008): "SIOSE, a successful test bench towards harmonization and integration of land cover /use information as environmental reference data", The International Archives of the Photogrammetry, Remote Sensing and Spatial Information Sciences, Vol. XXXVII, Part B8, pp. 1.159-1164. [Consulta: 10-03-2017]. Disponible en http://www.isprs.org/proceedings/XXXVII/congress/8 pdf/11 WG-VIII-11/28.pdf

Vidal-Macua, J. J.; Zabala, A; Ninyerola, M. y Pons, X. (2017): "Developing spatially and thematically detailed backdated maps for land cover studies", International Journal of Digital Earth, 10:2, pp. 175-206. 